\title{
A THEOREM ON FACTORIZATION*
}

BY D. N. LEHMER

In a note in this Bulletin $\dagger$ I observed that if $R=p q$ is the product of two odd factors whose difference is less than twice the fourth root of $R$ then the factors of $R$ are obtainable directly from the expansion of $R^{1 / 2}$ in a continued fraction. This theorem comes from the fact that in view of a theorem due to Lagrange, $(p-q)^{2} / 4$ will appear as a denominator of a complete quotient in that expansion, and that therefore the diophantine equation $x^{2}-R y^{2}=(p-q)^{2} / 4$ will have the integral solution $x=\frac{1}{2}(r+q), y=1$.

The object of the present note is to point out that the method is of much wider application than the above statement would indicate. For consider the identity

$$
\left(\frac{m p+n q}{2}\right)^{2}-\left(\frac{m p-n q}{2}\right)^{2}=m n p q .
$$

From this it appears that if $m n$ is a square and if $m$ and $n$ are both odd or both even, we will have an integral solution of the equation

namely

$$
x^{2}-R y^{2}=\frac{1}{4}(m p-n q)^{2},
$$

$$
x=\frac{1}{2}(m p+n q), \quad y=(m n)^{1 / 2} .
$$

By Lagrange's theorem, therefore, if $m p-n q<2 R^{1 / 4}$ one of the denominators in the expansion of $R^{1 / 2}$ will certainly be $(m p-n q)^{2} / 4$ and since the numerator of the preceding convergent will be $(m p+n q) / 2$ these two numbers will serve to furnish the factors $p$ and $q$ of $R$. We have then the following theorem.

* Presented to the Society, San Francisco Section, October 30, 1926.

† Vol. 13 (1906-7), p. 501. Translated in Sphinx-Oedipe, 1911. Given also in Kraitchik's Recherches sur la Théorie des Nombres, p. 73. 
THEOREM. If $R=p q$ is the product of two odd factors, and if two numbers $m$ and $n$, both even or both odd, are obtainable such that their product is a square and also such that $m p-n q<2 R^{1 / 4}$ then the continued fraction for $R^{1 / 2}$ will furnish without trial the factors $p$ and $q$ of $R$.

It should be noted that if the difference $m p-n q$ is less than the fourth root of $R$ the restriction that $m$ and $n$ be both even or both odd may be disregarded, for in that case $2 m$ and $2 n$ are suitable multipliers. Also it is worth noting that the square denominator will appear in the complete quotient when the denominator of the preceding convergent is $(m n)^{1 / 2}$. This means that in the original theorem the desired square is under the third complete quotient.

An example will indicate the method of attacking a number by this method. Let $A=1564,08789$. The square root expansion gives the following series of denominators for the complete quotients: $1,8753,15013,3740,529, \cdots$, the partial quotients being $12506,2,1,6,47, \cdots$. The convergent preceding the complete quotient with the square denominator 529 is found to be $250127 / 20$. We have then

$$
(m p+n q) / 2=250127, \quad(m p-n q) / 2=23 .
$$

Whence

$$
m p=250150, \quad n q=250104 .
$$

Since, now, $p q=A$ and $m n=20^{2}=400$ it is easily found that $p=31263, q=5003, m=8, n=50$. The success of the method was due to the fact that the difference $m p-n q=46$, which is less that $2 A^{1 / 4}=222$. In this example also we see that $36 p$ $-225 q=207$, which is also less than 222; but since here the values of $m$ and $n$ differ in parity, these values will not appear in the expansion. Similarly the difference $m p-n q=23$ for $m=4, n=25$, and $m$ and $n$ being different in parity these values will also not appear in the expansion. But since 23 is less than $A^{1 / 4}$, we will have $2 m$ and $2 n$ for suitable values, and these are indeed the ones that do appear.

The University of California 